\title{
Drift Theory and Plate Tectonics: A Case of Embedding in Geology
}

\author{
María Caamaño-Alegre ${ }^{1}$
}

(C) Springer Science+Business Media Dordrecht 2016

\begin{abstract}
The purpose of this paper is to elucidate the semantic relation between continental drift and plate tectonics. The numerous attempts to account for this case in either Kuhnian or Lakatosian terms have been convincingly dismissed by Rachel Laudan (PSA: Proceedings of the Biennial Meeting of the Philosophy of Science Association. Symposia and Invited Papers, 1978), who nevertheless acknowledged that there was not yet a plausible alternative to explain the so called "geological revolution". Several decades later, the epistemological side of this revolution has received much attention (Ruse in The darwinian paradigm, essays on its history, philosophy and religious implications. London, Routledge, 1981/1989; Thagard in Conceptual revolutions. Princeton University Press, Princeton, 1992; Marvin in Metascience 10:208-217, 2001; Oreskes in Plate tectonics: an insiders' history of the modern theory of the earth. Westview Press, Boulder, 2003), while the semantic relation between drift theory and plate tectonics has remained mainly unexplored. In studying this case under a new light, the notion of embedding, as distinguished from other sorts of intertheoretical relations (Moulines in Cognitio Humana-Dynamik des Wissens und der Werte. XVII, Institut für Philosophie der Universität Leipzig, Leipzig, 1996, Time, chance, and reduction: philosophical aspects of statistical mechanics. Cambridge University Press, Cambridge, 2010, Metatheoria 1(2):11-27, 2011), will have a particular significance. After formally analyzing the relationship between continental drift and plate tectonics, it will become evident that the models of drift theory are part of the models of plate tectonics, thereby fulfilling the conditions for embedding. All theoretical concepts from drift theory are presupposed in some theoretical concepts from plate tectonics, and all empirical concepts of the former are shared by the latter. Furthermore, all the successful paradigmatic applications of continental drift are also successful applications of plate tectonics. As a consequence, under the label "geological revolution", we actually find a salient historical case of cumulative progress across theory change.
\end{abstract}

María Caamaño-Alegre

mariac@fyl.uva.es

1 Department of Philosophy, University of Valladolid, Valladolid, Spain

Published online: 11 November 2016 
Keywords Embedding $\cdot$ Geological revolution $\cdot$ Plate tectonics $\cdot$ Continental drift $\cdot$ Partial substructures

\section{Introduction}

The current idea of scientific development is still strongly inspired by T. S. Kuhn's distinction between normal science and revolutionary science (Kuhn 1962). Without disputing the relevance of such dichotomic distinction to understand the sharp differences between continuous and discontinuous periods of scientific advancement, it may be helpful to emphasize the significance of a peculiar historical episode that has remained misidentified as a result of exploring the history of science through a Kuhnian lens. As explained in the next section, the numerous attempts to account for the relation between continental drift and plate tectonics in Kuhnian terms have been convincingly dismissed by several authors, who nevertheless have not yet supplied a plausible alternative to explain the so called "geological revolution". The purpose of this work is to show the relevance of the notion of embedding for elucidating the above historical case. This mathematical, model-theoretic notion was originally used in the philosophy of empirical science by Suppes and then further applied mainly by semanticist philosophers like Van Fraassen (1980, 42, 43), according to whom it requires the inclusion of each model of a theory into a model of another theory-every model of $T_{1}$ would thus be identified with a substructure of a model of $\mathrm{T}_{2}$. Even if the above characterization captures the core of the notion of embedding, the one that I will apply has been provided by Moulines (2011), who introduces the key notion of echeloned partial substructure and thereby makes it possible to reveal the mode of preservation of partial substructures. By studying this historical case under a new light, the very notion of embedding will become clearer and its explanatory power more obvious.

As pointed out by Moulines, cases of embedding should be distinguished from three other kinds of diachronic developments in science, such as evolution, replacement and crystallization (1996, 2010, 41-42; 2011, 13-17; 2014, 1507-1511). Normal science-as well as the Lakatosian notion of research program- roughly corresponds to what Moulines calls "evolution", since in both cases a theoretical network (or theory-net, for short) evolves in time by adding or removing from the network some specialized theories (theoryelements, in what follows), without losing its identity, which is determined by an unchanged initial or basic theory-element. ${ }^{1}$ The development of Newtonian mechanics from the end of the seventeenth century until the beginning of the last century is often mentioned as a case in point. Revolutionary science, on the other hand, can be equated with

\footnotetext{
1 In structuralist literature (Balzer et al. 1987, 167-177), the notion of a theory-net is defined by establishing two conditions: 1) that there should exist a finite non-empty set of theory-elements $\mathrm{T}^{\mathrm{E}}$ and a specialization relation $\sigma ; 2$ ) that the specialization relation be restricted to the set $\mathrm{T}^{\mathrm{E}}$. Given that the definition of a theorynet presupposes that of specialisation, the principle defining features of this should be mentioned, even if in passing. They are as follows: a) equality between the classes of potential models and partially potential models of the respective related theory-elements; and, b) the inclusion of the current class of models, the class of constraints, the class of links and that of intended applications of the (resulting) specialized theory, respectively, in the class of actual models, the class of constraints, the class of links, and that of the intended applications of the theory that is specialized. Expressed in other terms, two theory-elements that are related by means of a specialization relation will share their conceptual apparatus, while they will diverge with regard to the scope of their laws and, consequently, with regard to the extension of their classes of intended applications, since the theory-element that specializes restricts the laws and the empirical scope of the specialized theory-element.
} 
Moulines' notion of replacement, which refers to cases where a theory is completely replaced by another one with similar intended applications, although very different from a conceptual point of view. The superseding of Ptolemaic astronomy by Copernican astronomy or that of the phlogiston theory of combustion by the oxygen theory are usually presented as clear examples of replacement. Unlike other sorts of diachronic developments that clearly exhibit either a continuous character or a discontinuous one, crystallization occurs when continuous and discontinuous aspects are combined in the emergence of a complex, fully developed theory (Moulines 2010, 142). According to the reconstruction by Moulines (2011), Clausius's Phenomenological Thermodynamics would be a case of crystallization. Among the plausible not yet reconstructed cases $(2011,15)$, he mentions the emergence of Ptolemaic astronomy (s. III a.C.-s. II d.C.), Cartesian mechanics (1630-1670), and Mendelian genetics (1865-1905).

Furthermore, Moulines noted that there is possibly a second Kuhnian sense of revolutionary science- hardly discussed by Kuhn himself and most frequently overlooked by his critics- ${ }^{2}$ which points to cases of cumulative progress where the models of a previous theory are (approximately) embedded into the models of a more complex theory, hence the label "embedding". The relation between Keplerian planetary theory and Newtonian mechanics, as well as that between Cartesian collision mechanics and the latter, provide two historical illustrations of embedding, where a new theory, with a wider scope of application, approximately includes the content of an old theory (Balzer et al. 1987, chapters VI-VII). This kind of advancement, although very different from the one characteristic of normal science, is still markedly continuous in the sense that it does not entail discarding the earlier theory, but rather subsuming it under the latter one. Interestingly, the case of embedding under study here is not mentioned among the plausible not yet reconstructed cases suggested by Moulines $(2011,16)$, which would include the embedding of the wave optics into Maxwell's electrodynamic theory, and of the latter into the special theory of relativity, or the embedding of the Darwinian theory of evolution into the synthetic theory of evolution. As shown in Sect. 4, however, the emergence of plate tectonics nicely fits the conditions defining embedding.

It is interesting to take a moment and notice how Kuhn himself discusses, and then disregards as irrelevant, the possibility of knowledge accumulation from old paradigms to new ones. After stressing that the assimilation of either a new sort of phenomenon or a new scientific theory entails the rejection of an older paradigm, he denies that this can be explained by virtue of the logical structure of scientific knowledge. He then proceeds to briefly consider possible cases in which the acceptance of a new theory would not prompt the rejection of the preceding one:

"In principle, a new phenomenon might emerge without reflecting destructively upon any part of past scientific practice. (...) a new theory does not have to conflict with any of its predecessors.

(...) Nevertheless, despite the immense plausibility of that ideal image, there is increasing reason to wonder whether it can possibly be an image of science. After the pre-paradigm period the assimilation of all new theories and of almost all new sorts of phenomena has in fact demanded the destruction of a prior paradigm and a consequent conflict between competing schools of scientific thought. Cumulative acquisition of unanticipated novelties proves to be an almost non-existent exception to the rule of scientific development. The man who takes historic fact seriously must

\footnotetext{
${ }^{2}$ One of the few places (if not the only one) where Kuhn elaborates on this matter is 1962/1970, 95-97.
} 
suspect that science does not tend toward the ideal that our image of its cumulativeness has suggested. Perhaps it is another sort of enterprise" (Kuhn 1962/1970, 95-96).

Thus, according to Kuhn, there is no intrinsic logical constraint on scientific knowledge requiring that new paradigms conflict with old ones. Yet, as a matter of historical fact, the usual dynamics of scientific practice renders conflict between successive paradigms as the rule and lack of conflict as "an almost non-existent exception". Among the exceptional cases, he mentions quantum mechanics, which mainly accounts for new phenomena just replacing ignorance, and the theory of energy conservation, which provides a higher level theory connecting a group of lower level theories. Only the former example would provide a case of embedding in the sense previously discussed.

The present analysis of the geological revolution in terms of embedding casts some doubts on the accuracy of the above Kuhnian view, undermining the idea that cases of embedding constitute extremely rare episodes of scientific development. But before turning to the case of geology, a more detailed characterization of embedding is provided. A historical sketch of the emergence of plate tectonics and an overview of the philosophical controversies around it are provided in the third section. The fourth section is devoted to the analysis of the semantic relation between continental drift and plate tectonics, one that, as it is shown there, meets the conditions for embedding. The philosophical consequences that can be drawn from this analysis are fully elaborated in Sects. 4 and 5.

\section{The Meta-theoretical Notion of "Embedding"}

Before presenting Moulines' definition of embedding, let us clarify some basic structuralist notions. From the structuralist standpoint, a scientific theory is not just constituted by a class of models (i.e., sequences of set-theoretical entities) satisfying a set-theoretical predicate, but by different classes of models, hierarchically organized according to the level of complexity, and satisfying a correspondingly complex set-theoretical predicate (Moulines 2002, 4-5). Two main classes of models are distinguished: on the one hand, the class of potential models (' $\mathrm{M}_{\mathrm{p}}$ ', from now on), which just satisfy the theory's frame conditions, and, on the other hand, the actual models (' $\mathrm{M}$ ' in what follows), which satisfy both the frame conditions and the substantial/theoretical laws (Balzer et al. 1987, 2-6). Frame conditions just supply the conceptual machinery or descriptive resources of a theory, that is, they set the formal properties of the scientific concepts employed in a theory (i.e. of each domain or relation included in the theory). The empirical substructures of $\mathrm{M}_{\mathrm{p}}$ form the class of a theory's partial potential models (' $\mathrm{M}_{\mathrm{pp}}$ ' from now on). Partial potential models play a decisive role in the empirical interpretation and application of the potential models in which they are included (Balzer et al. 1987, 277). Since usually a theory is primarily directed towards the empirical substructures of $\mathrm{M}_{\mathrm{pp}}$, within the structuralist approach such specially relevant subset has been differentiated from the rest of empirical substructures and characterized as T's "intended applications" ('I', in what follows). As this very label suggests, I has a predominantly pragmatic nature, for it includes those empirical substructures that are targeted by the scientific community endorsing the theory. The distinction between $\mathrm{M}_{\mathrm{p}}$ (theoretical structures) and $\mathrm{M}_{\mathrm{pp}}$ (empirical substructures) is drawn on the basis of another distinction, that between T-theoretical concepts and T-nontheoretical concepts. T-theoretical concepts can only be determined by applying T's actual models (corresponding to the substantial laws), T-non-theoretical models, on the other 
hand, are determined by models coming from outside of $\mathrm{T}$, typically by the actual models of other underlying (presupposed) theories included (Balzer et al. 1987, 62-67, 73-77, Moulines 2002, 7-8).

In a paper from 2011, Moulines introduces the key notion of echeloned partial substructure in order to develop a more fine grained analysis of the piecemeal fashion in which the models of a new theory are constructed. As will become clear in the forthcoming sections, such notion proves very helpful in accounting for the embedding of drift theory into plate tectonics, a case in which, according to the present study, preservation of partial substructures of theoretical models plays a crucial role.

The development of new formal resources to provide a more fine-grained differentiation between separate constituents of models goes hand in hand with the advances in explaining the nature of inter-theoretical relations. Moulines' technical notion of echeloned partial substructure is a case in point $(2011,18 ; 2014,1511)$, since it has already been proven applicable not only to the crystallization of Clausius's Phenomenological Thermodynamics (2011, 19-21), but also to cases of radical incommensurability between theories connected by an ontological non-reductive relation (Falguera and Donato Rodríguez 2015, 15). Following Moulines' formal account of embedding, which he defines by using the notion of echeloned partial substructure, we can start by clarifying the standard notion of partial substructure.

Let $S=\left\langle A_{1}, \ldots, A_{\mathrm{n}}\right\rangle$ be a structure with $m$ domains $D_{1}, \ldots, D m$ and $n-m$ relations (with $n>m) R_{m+1}, \ldots, R_{n}$. Let $S^{*}$ be a structure. Let $A$ be a domain or a relation.

Definition 1.1 $A \hat{\in} S$ iff $\exists i\left(1 \leq i \leq n \wedge A=A_{i}\right)$.

" $A \hat{\in} S$ " is read as: " $A$ is a component of $S$ ". 3

Definition 1.2 $S^{*} \hat{\in} S$ iff $\forall A \hat{\in} S^{*}: A \hat{\in} S$.

" $S$ * $\hat{\in} S$ " is read as " $S$ " is a partial substructure of $S$ ". Trivially, any component of $S$ can be regarded as a substructure of $S .^{4}$

Simply put, the idea of an echeloned partial substructure is that of a relation of set theoretical construction between substructures, so that one of them results from applying certain set-theoretic operations over the components of the other. The latter is hence regarded as an echeloned partial substructure of the former, satisfying the following definitions:

Definition 1.3 $\Theta$ is the set-theoretic operation consisting in successively applying "Pot" (power set) and " $x$ " (cartesian product) to some previously given set(s) a finite number of times to, always beginning with Pot.

Definition 1.4 $\mathrm{A}$ is an echelon-set over $B_{1}, \ldots, B_{m}$ iff $A \in \Theta\left(B_{1}, \ldots, B_{m}\right)$.

Definition 1.5 $S$ is an echeloned partial substructure of $S^{*}\left(S \eta S^{*}\right)$ iff $\forall S_{i}\left(S_{i} \hat{\in} S\right) \exists S_{k}^{*}\left(S_{k}^{*} \hat{\epsilon}\right.$ $\left.S^{*}\right): S_{i} \in \Theta\left(S_{k}^{*}\right)$.

\footnotetext{
3 The reader should note that the symbol ' $\hat{\epsilon}$ ' expresses a relation between a component of a structure and the structure itself, and so it is not to be confused with the element-of symbol ' $\epsilon$ ' used later, which expresses a relation between an element and a set.

${ }^{4}$ Here we are assuming the notion of substructure that is standard in model theory. Under this characterization, a structure $S$ is a substructure of other $S$ ' when the domains of $S$ are proper or improper subsets of the domains of $S^{\prime}$, and, therefore, the relations of $S$ are restrictions over the relations of $S$ '.
} 
We can now turn to the formal definition of embedding, which first requires defining the auxiliary notion of "success" (Moulines 2011, 24; 2014, 1517):

Definition 2.1 Let $m$ be an actual model of a theory-element $T_{i}$ from the net $N$. Then, $m$ is a "successful model" in $N$ iff there exists an intended application $y$ of $T_{i}$ such that $y=r(m){ }^{5}$ Similarly, $y$ is a successful application iff there exists an actual model $m$ of $T_{i}$ such that $y=r(m)$.

Definition 2.2 The Theory-net $N$ is embeddable into net $N^{*}$ iff.

1. Any successful model in $N$ is an (approximative) echeloned partial substructure of some successful model in $N^{*}$;

2. Any successful intended application in $N$ is an (approximative) echeloned partial substructure of some successful intended application in $N^{*}$.

According to this definition, the embedding of a theory-net into another involves the fulfilment of two conditions: first, for every successful model in $N$ there is a set-theoretic construct in $N^{*}$ that results from performing operation $\Theta$ over the components of such model and constitutes a successful model in $N^{*}$; second, for every successful application in $N$ there is a set-theoretic construct in $N^{*}$ that results from performing operation $\Theta$ over the components of such application and constitutes a successful application in $N^{*}$. Given that the reconstruction presented here is restricted to the basic theory elements of two successive geological theories, I will skip the details regarding theory-nets. The former conditions for embedding will thus be examined only with regard to successful models and applications of the corresponding theory-elements.

\section{A Historical Sketch of the Emergence of Plate Tectonics and the Philosophical Controversies Around it}

The so called "geological revolution" took place towards the middle of the twentieth century, after a long journey of accumulation of empirical data (Thagard 1992, 163, 171-190, Oreskes 2003, 13-27). Geological anomalies like that of coastlines' fit remained unexplained by the theory of Earth's contraction and could only be later explained by Wegener's theory of continental drift, which nonetheless, as often pointed out by historians, did not supersede the contraction theory until it was subsequently modified and completed by plate tectonics.

According to the standard literature on the subject (Laudan 1978; Cohen 1985/2001; Thagard 1992; Marvin 2001; Oreskes 2003), when Alfred Wegener presented his conjecture regarding continental drift in 1915, the predominant view was that the solid Earth had a stable nature and that actual geological accidents like mountains had been caused by contraction due to the decrease in temperature. Yet, the jigsaw-puzzle fit of the continental edges was a well known fact as early as the 16th century, and such fact, together with others established in the 19th century from paleontological and paleoenvironmental research, were the ones that led Wegener to develop the theory of continental drift. More precisely, he relied on observations regarding: (1) the fit of the continents' edges, (2) the correlation of fossil plants and animals as well as of rock strata across oceans, and (3) the presence of cold climate indicators (such as ancient glacial deposits) near the equator, and

\footnotetext{
5 Function ' $r$ ' assigns to every actual model ' $m$ ' the corresponding partial potential model ' $y$ ' that results from cutting the T-theoretical concepts from ' $\mathrm{m}$ '.
} 
the presence of warm climate indicators (such as limestones, laterites and coals) near the poles. Wegener explained all these facts by supposing that, originally, all continents were together forming a single supercontinent (Pangaea) and that, through time, they moved away from each other thereby shifting their position. According to his theory, mountain formation is caused by the resistance of cool oceanic crust to continental movement.

It has been frequently emphasized by historians and philosophers dealing with the geological revolution that Wegener's theory faced a strong opposition by most geologists of that period, and it was never fully accepted by the scientific community (Frankel 1979, 49-52; Ruse 1981-1989, 73-74, 85-87; Cohen 1985/2001, 446, 464-465; Marvin 2001, 213-215; Oreskes 2003, 10-12). The replacement of the contraction theory did not happen until the $60 \mathrm{~s}$, when plate tectonics developed and received strong support from many mutually independent sources, such as oceanographic, paleomagnetic, and seismic evidence. These kinds of evidence would make it possible to develop an empirically sound account of continental movement, one that not only covered the main explanatory gap in the previous account-namely, the causal mechanism of such motion- but provided a wide variety of independent and strong empirical support for the postulated mechanism. The key importance of the new evidence is stressed by M. Ruse's remark that:

“(...) although to the lay-person fitting together the continents is the key point in the new geology - to many of the new geologists themselves it is somewhat peripheral.

What really counts is the newness of the ocean beds, and the magnetic reversals" (1981-1989, 83).

Let us recall that it was not until quite recently (i.e. from the nineties) that scientists could find some direct evidence concerning the motion of continents, and, for most of the twentieth century, the predictive capacity of geology remained quite limited, so refutation of geological theories by means of their false predictions was not a serious option. Hence, the current theory of plate tectonics was developed between 1962 and 1968 on the basis of several independent observations from sea floor and earthquakes, originally collected by geophysicists who were not involved in the development of this theory. By the end of the fifties, there was no doubt that the Earth's magnetic field had changed repeatedly throughout time, as both the remanent magnetism detected in sedimentary continental rocks and the magnetic anomalies observed round the mid-ocean ridges clearly suggested those magnetic reversals (Oreskes 2003, 19-20). The drift of continents thereby became the most plausible explanation for a variety of independently established facts. The new results from oceanography that were now available to geologists also provided evidence for Harry H. Hess' hypothesis that the sea floor was spreading. The distinctive striped pattern of magnetized basaltic rocks on both sides of the mid-ocean ridges, together with the transfer of heat detected in those areas, led to the conclusion that lava was continuously flowing from canyons located along the crest of the ridges (Oreskes 2003, 21-23). Finally, the accurate determination of earthquakes' locations and directions displayed a pattern resembling moving blocks. All this combined evidence supported the conjecture that both continents and sea floors stand on moving plates whose dynamics manifests itself in the phenomena of sea floor spreading and destruction. Such conjecture was independently developed by several geologists, like J. Tuzo Wilson, Daniel P. McKenzie together with Robert L. Parker, and Jason Morgan, providing a synthetic picture of crustal motions as rigid body rotations on a sphere (Oreskes 2003, 25-27). Mountains, on the other hand, were now understood as geological formations resulting in some cases from the pressure and friction between colliding plates and, in some other cases, from collision between continents and island arcs, oceanic plateaus and microcontinents (Frisch et al. 2011, 
149-158). In all these cases, a very gradual process of subduction of continental or oceanic crust originated the later collisions. The overall picture resulting from this decades-long gathering of evidence is presented by Oreskes as follows:

"A global picture now emerged. Oceans split apart at their centers, where new ocean floor is created by submarine volcanic eruptions. The crust then moves laterally across the ocean basins. Ultimately, it collides with continents along their margins (edges), where the ocean crust sinks underneath, back into Earth's mantle. As it does, it compresses the continental margins, generating folded mountain belts and magmas that rise to the surface as volcanoes, and deep earthquakes as the cold, dense ocean slab sinks farther and farther back into the earth" (2003, 26-27).

Although the predominant tendency among historians and philosophers was to provide a Kuhnian explanation of the geological developments that occurred over the twentieth century, this sort of account has been criticized on different grounds. When Rachel Laudan's critical paper “The Recent Revolution in Geology and Kuhn's Theory of Scientific Change" was published in 1978, the standard view that changes taking place in geology from the sixties amounted to a Kuhnian revolution had been hardly questioned. On this matter, she mentions some historians like J. Tuzo Wilson, Ursula Marvin, Allan Cox or Arthur Hallam, who, although more cautious than the others, also argued that the nonlinear development of geology fitted the pattern of a revolution in the Kuhnian sense. It should be noticed, however, that this non-linear nature of geological research, was only broadly characterized and described in different terms by different authors, partly depending on what features, among the ones initially pointed out by Kuhn as typical of a change of paradigm, were taken into account. Even if some authors endorsed the view there is a conceptual break between pre- and post-plate tectonic theories, in most cases, the corresponding analyses were conceptually informal and usually included a reference to a wide variety of heterogeneous features such as changes of methods, shift of standards of evidence, or the sudden conversion of scientists. According to Laudan, the main criticism to the standard interpretation came from David Kitts, who argued that the existence of shared basic physical principles underlying competing geological theories is not compatible with a Kuhnian revolution, which would require the replacement of a comprehensive paradigm. As she notes, however, Kitts' reading of Kuhn seems unconvincing for several reasons, not only because some classical Kuhnian examples, like the Darwinian revolution or the Lyellian revolution, do not imply any replacement of fundamental physical or chemical principles, but also because Kuhn himself acknowledged that the scope of revolutions could be wider or narrower (1969/1970, 198-202). ${ }^{6}$ In arguing for the quality of the evidence supporting geological theories as the key aspect involved in their career, Laudan dismisses the idea that their possible conflict with physical laws or their lack of an explanatory physical mechanism were essential in that regard $(1978,229-232){ }^{7}$ She concludes that the emergence of plate tectonics was neither a Kuhnian revolution- since there was no incommensurability between this theory and drift theory, nor a Lakatosian evolution, as suggested by Frankel (1979)—since no stability of a hard core seems

\footnotetext{
${ }^{6}$ Furthermore, in Kuhn's later proposal (1983, 670-1), he explicitly characterizes the notion of incommensurability as a local or partial relation between theories.

7 By stressing the importance of evidence quality as the driving force in the career of geological theories, she therefore also opposes the popular view put forward by Stephen Jay Gould (1977), which implied that the initial rejection of drift theory was due to a lack of an adequate mechanism to move continents through a static ocean floor.
} 
compatible with the new ontological commitments entailed by plate tectonics, like the very notion of tectonic plate or that of sea floor spreading (Laudan 1978, 235-236). More broadly, she concedes that understanding the emergence of plate tectonics remains an open question.

Laudan rightly questions the standard approach to twentieth century geology based on the Kuhnian concept of scientific revolution, as well as the one inspired by Lakatos' ideas. However, since she is most interested in explaining the epistemological side of the historical career of drift theory, she focuses on issues related to evidence and the corresponding decisions of acceptance or rejection taken by the scientific community, rather than on the conceptual implications of the relation between drift theory and plate tectonics, which is the focus of the current analysis. Several issues, thus, still pose some problems for the accounts of the development of geology available to present. Many of such problems arise from a lack of detail in analyzing the conceptual constituents of theories. In fact, none of the wide and heterogeneous set of features commonly regarded as characteristic of a change of paradigm in the Kuhnian sense-which would include replacement of general disciplinary principles, shift of methodological and evidential standards or sudden conversion- is the essential feature consistently emphasized by Kuhn in his writings. The essential feature to which he draws attention is the conceptual incompatibility or incommensurability between rival theories. It should be remembered that the notion of incommensurability was introduced simultaneously and independently by Kuhn (1962/1970), and Paul K. Feyerabend (1962/1981), with the aim of characterizing relations between rival scientific theories. Both authors coincide in presenting it as a radical form of conceptual disparity between theories that are applied to the same field of research. The radicality of conceptual disparity was due not only to the fact that theories would be made up of different concepts, but also to the fact that the conditions for the formation of these concepts would be incompatible. In accordance with both authors, this becomes apparent in the incompatibility between the rules of use for the terms that transmit these concepts. Incommensurability between rival scientific theories would be patent not only in the impossibility of mutually translating the languages that express these theories, but also in the impossibility of expanding one of these languages in order for it to incorporate the other.

The question as to whether the aforesaid problem may have arisen between geological theories has remained unclear in the available discussions on the subject. One exception is worth mentioning: Thagard's approach to the semantic side of the revolution in geology, where he certainly applies some central Kuhnian notions, like the idea that changes in taxonomic categories are characteristic of scientific revolutions (1992, 7-8, 160-170). His argument that the emergence of plate tectonics involved a dramatic reorganization of the kind-hierarchy and part-hierarchy developed from drift theory is nevertheless questionable. According to his account, the new concept of sea floor spreading was crucial in the above reorganization, which concerned continents and sea floors, more specifically involving their reconceptualization not only as different kinds of surface but also as parts of plates, instead of parts of the earth's crust $(1992,182)$. Despite Thagard's careful attempt to provide a Kuhnian picture of the development of plate tectonics, there is one important element missing, and that is the semantic incommensurability between both theories. Regardless of whether one endorses the Kuhnian view on scientific revolutions-and therefore of how one understands the label "scientific revolution", what is clear is that there is no conceptual incompatibility between the central notions of both theories. In fact, the taxonomic changes identified by Thagard result in an addition of properties ascribed to continents and sea floors, rather than in a conflicting conceptualization of these geological 
units. Contrary to what Thagard seems to suggest, considering continents and sea floors as parts of plates does not exclude but rather presupposes considering them as parts of the earth's crust. In short, kind-hierarchy and part-hierarchy developed from drift theory is presupposed in the corresponding hierarchies from plate tectonics. The acknowledged differences between continents and sea floor certainly increase with the emergence of the latter, but the newly determined properties mainly contributed to develop a more detailed account of the Earth's crust, thereby supplementing the account of the crust provided by drift theory. Continents and sea floors were certainly assumed to be similar parts of the crust in Wegener's theory and very dissimilar parts of the crust in Hess' theory, however, this assumption was not essential to drift theory, since the assumed similarity played no explanatory role in this theory.

Arguments similar to the previous ones have been put forward by Laudan (1978, 228, 235) in rejecting Cox's view that there is incommensurability between drift theory and plate tectonics. Although the analysis offered in the next section is just meant to clarify the conceptual relationship between both theories, an intuitive application of the notion of incommensurability clearly supports Laudan's view. While the difference between the vertical movement of continents postulated from the contraction theory and the lateral movement postulated from drift theory could represent a case of incommensurability, no conceptual incompatibility of this kind is detectable between drift theory and plate tectonics, since both theories share the assumption about lateral continental movement.

On the contrary, the idea that there is a constitutive link between continental drift and plate tectonics, despite the historical neglect of the former over decades, can be found in Oreskes' remarks that:

"Continental drift was not accepted when first proposed, but in the 1960s it became a cornerstone of the new global theory of plate tectonics. The motion of land masses is now explained as a consequence of moving "plates"-large fragments of the earth's surface layer in which the continents are embedded" (2003, 3).

It is important to notice, however, that the motion of continents was not part of the intended explanandum of plate tectonics but rather part of its explanans, since both theories rely on such motion to explain some other facts that are included in their shared intended explananda, like mountain formation. Furthermore, the movement of continents was not taken for granted as an empirical fact by the time plate tectonics emerged, it was rather part of a conjecture intended to explain some new well-established facts, like sea floor spreading and destruction. The intuitive idea that a theory provides the explanandum for another theory is captured by the structuralist notion of theoretization (Balzer et al. 1987, 251), the latter referring to that intertheoretical relation where the empirical concepts of $T^{*}$ (i.e. $\mathrm{T}^{*}$-non-theoretical concepts) are concepts belonging to $\mathrm{T}$ ( $\mathrm{T}$-theoretical or $\mathrm{T}$-nontheoretical). Interestingly, as I will show later, plate tectonics and drift theory do share some empirical concepts, hence meeting the minimal condition for what Balzer et al. call "weak theoretization", namely, that some of $\mathrm{T}^{*}$-non-theoretical concepts come from $\mathrm{T}$. Yet, this only means that both theories overlap in their respective explananda, not that one constitutes that explanandum for the other, which would require what they call "strong theoretization"- -where all of $\mathrm{T}^{*}$-non-theoretical concepts come from $\mathrm{T}$. Further discussion of this issue is provided in Sect. 4.3.

In short, we have ruled out three different alternative ways to understand the relationship between both theories, namely, a Kuhnian revolution connecting incommensurable theories, a Lakatosian evolution from a stable hard core, and a theoretization of 
continental drift from plate tectonics. In the next section, the relation between both theories is interpreted as a case embedding.

\section{The Embedding of Continental Drift into Plate Tectonics}

The remaining of the paper is focused on the relation between continental drift theory and plate tectonics. In the next section I will formally analyze the main three classes of models (i.e. potential, actual, and partial) constitutive of continental drift and plate tectonics. By clarifying the structure of each theory, it will become evident that the models of drift theory are part of the models of plate tectonics, thereby fulfilling the conditions of Definition 2.2. This constitutive relation holds for each kind of model, that is, between the respective potential, actual and partial models of both theories. All theoretical concepts from drift theory are presupposed in some theoretical concepts from plate tectonics. The very concept of continental drift, for instance, is implied by the concept of tectonic drift. On the other hand, their respective applicative domains and paradigmatic applications also present a significant overlap, since both theories share most empirical concepts, i.e., those inherited from either some pre-theoretical notions, or some well-established, commonly accepted theories.

For the sake of simplicity, I will not reconstruct here two of the characteristic components of the above theories, to wit, their respective inter-theoretical links-i.e. crossconnections between the models of different theories- and constraints-i.e. cross-connections between the models of one and the same theory (Moulines 2002, 7). Instead of reconstructing the links that are necessary to define some non-theoretical concepts of each theory, I shall just mention some of the different theories on which the determination of these concepts depends. The same goes for constraints on the properties that the targeted systems should preserve at different applications of drift theory and plate tectonics (Balzer et al. 1987, 40-47). I shall here confine myself to mentioning a main non-theoretical constraint shared by both theories, one usually referred to as "principle of isostasy" (Thagard 1992, 166). This geophysical principle asserts that, if not disturbed, the crust is in gravitational equilibrium, there being equal forces holding it up and pulling it down. The buoyancy force of different pieces of crust floating on denser strata below would counteract gravity. Accordingly, in order for a piece of crust to be part of different models from either theory, it should be in isostatic equilibrium, so that any vertical force acting in one direction would be compensated by other vertical force acting in the opposite direction.

\subsection{Continental Drift Theory ('DRIFT' in what follows)}

\subsubsection{DRIFT's Conceptual Framework}

Let us start by examining those basic domains and relations that determine DRIFT's class of potential models, thereby constituting its basic conceptual framework.

DRIFT's basic domains are represented by $L$, which is a set of volumes of lithosphere, $T$, which is interpreted as a set of temporal instants, and $S$, which represents a set of spatial regions. These three domains are finite, non-empty sets, and $T=\left\{t_{1}, t_{2}\right\}$.

As for the non-basic domains or subsets of $L$, we have $U$, which is a set of volumes of earth's crust, $C$, which is interpreted as being a set of volumes corresponding to continents, $M$, interpreted as a set of volumes corresponding to mountains, and $F$, equated with a set of 
volumes corresponding to sea floors. All of them are non-empty subsets of $U$, and $F \cap$ $C=\varnothing$.

Passing now to DRIFT's relational concepts, we find $P$, which is the mereological relation of parthood between volumes of crust, and does not have a functional character. $P$ $\subseteq U \times U$, and it determines a partial, non-strict order. The movement of the continents is represented by means of the function $d$, under which each volume corresponding to a continent and a certain spatial region in $t_{1}$ have assigned the volume of a continent and a different spatial region in $t_{2}$ :

$$
d:\left(C \times S \times\left\{t_{1}\right\}\right) \rightarrow\left(C \times S \times\left\{t_{2}\right\}\right)
$$

The function $k$ determines the kinetic friction between volumes of continents and volumes of sea floors, by assigning to each couple of neighbouring continent and sea floor at a given time a friction coefficient: $k: C \times F \times T \rightarrow \mathrm{R}^{+}$

$I$ is the fit relation between volumes of continents and its character is not functional: $I \subseteq$ $C \times C$

The structures contained in DRIFT's class of potential models are tuples of the following kind: $<L, U, T, S, C, M, F, \mathrm{R}^{+}, d, k, P, I>$, which satisfy the above typifications and characterizations.

\subsubsection{DRIFT's Laws}

Apart from the axiom (1) stating that DRIFT's class of actual models is a subset of its class of potential models, there are three fundamental laws unfolding DRIFT's specific content.

Axiom (2) establishes that all continents existing today were part of the same continent at $t_{1}$. That is:

There is a $c^{\prime} \in C$ at $t_{1}$ such that for all $c \in C$ at $t_{2}: c P c^{\prime}$ at $t_{1}$

In axiom (3), continents' outline fit occurring at present is explained on the basis of a previous continental separation. Formally expressed:

There are some $c, c^{\prime}, \mathrm{c}^{\prime \prime} \in C$ such that: if $c P c^{\prime}$ ' and $c^{\prime} P c^{\prime}$ ' at $t_{1}$ and it is not the case that $c P c$ ' nor that $c$ 'Pc' at $t_{2}$, then $c I c$ ' at $t_{2}$

Axiom (4) states that the location of continents changes along time, which means that continents move with respect to each other. That is:

For all $c \in C$ : there is some $<c, \mathrm{~s}, t_{1}>\in \operatorname{Dom}(d)$ such that: $d\left(<c, \mathrm{~s}, t_{1}>\right)=<c$, s', $t_{2}>$ and $\mathrm{s} \neq \mathrm{s}$

Finally, the last law asserts that mountain formation depends on some kinetic friction taking place between continents and sea floors. Put formally:

Axiom (5) For all $m \in M, c \in C$ and $f \in F$ :

there is some $f \in F$ such that if $k\left(<c, f, t_{1}>\right)=i \neq 0$ and $k\left(<c, f, t_{2}>\right)=i^{\prime}$ with $i^{\prime}>i$, then $m P c$ or $m P f$ at $t_{2}$

\subsubsection{DRIFT's Empirical Framework}

Turning now to DRIFT's class of partial potential models, let us see what elements of the tuple representing the potential models should be removed given the fact that their determination necessarily involves DRIFT's fundamental laws. Such removal leads to the following kind of structure: 
$y$ is a partial potential model of the continental drift theory $\left(y \in \mathbf{M}_{\mathbf{p p}}(\mathbf{D R I F})\right)$ iff there exists an $x$ such that $x=<L, U, T, \mathrm{~S}, C, M, F, \mathrm{R}^{+}, d, k, P, I>\in \mathbf{M}_{\mathbf{p}}($ DRIFT) and $y=<L$, $U, T, S, C, M, F, \mathrm{R}^{+}, P, I>$.

The matter of theoreticity concerns $L, U, T, S, C, M, F, d, k, P, I$. The three basic sets, along with the four non-basic ones, and the (non-functional) relations $P$ and $I$ are DRIFTnon-theoretical, as they may all be determined without relying on DRIFT's fundamental laws. $d$ and $k$, by contrast, must be considered as DRIFT-theoretical given its dependence on these laws, more specifically on axioms (4) and (5). The notions of continental movement and kinetic friction between continents and sea floors can only be defined on the basis of DRIFT's postulation of such functions.

I shall now go on to examine the criteria for determining the functions and/or notions expressed by DRIFT-non-theoretical terms. The phenomena or entities to which these terms refer will be recognized, at least in part, by taking these criteria into consideration. The notion of lithosphere $(L)$ was first introduced by Joseph Barrell in 1914, with the publication of his article "The Strength of the Earth's Crust", and more than two decades later developed by Reginald Aldworth Daly in his Strength and Structure of the Earth (1940). The term lithosphere' referred to the Earth's strong outer layer, which was thought to surround a weaker, floating layer called 'asthenosphere'. Several striking gravity anomalies over continental crust were explained on the basis of these two notions. The determination of $T$ and $S$ respectively requires some type of chronometric and topological theory. As for $U, C, M$ and $F$, during the historical period in which DRIFT prevailed they were determined by principally applying pre-scientific notions, belonging to the sphere of ordinary knowledge. The notions of crust, continent, mountain and sea floor were introduced into geological theories as primitive terms, and therefore they were not defined in these theories. Each of these notions could only be determined on the basis of a disjunction of descriptions or predicates that express different properties: location, surroundings, material constitution, shape, etc. On the other hand, regarding DRIFT-non-theoretical relations, the use of some mereological theory is essential in the determination of the parthood relation $P$, and some cartographic studies are needed to determine the fit between volumes of continents $I$.

Continent and mountain formation were the principal phenomena dealt with by theories previous to DRIFT. In addition to the relation of fit between volumes of continents $I$, $C$ and $M$ are the main DRIFT-non-theoretical domains to be explained by the theory.

\subsection{Plate tectonics ('TEC' in what follows)}

\subsubsection{TEC's Theoretical Framework}

TEC includes all of DRIFT's basic and non-basic domains, therefore $L, U, T, S, C, M$, and $F$ are interpreted the same way as in DRIFT. There is a new non-basic domain added in TEC, namely, $O$, which is a subset of $L$ and is interpreted as a set of tectonic plates.

All of DRIFT's relational concepts are also included in TEC, with the addition of six more, namely, sea floor spreading, sea floor destruction, seismic activity, movement of the tectonic plates, kinetic friction between continents and that between tectonic plates. More precisely, $e$ is a function that determines the sea floor spreading by assigning to some volume of sea floor $f$ at $t_{1}$ a bigger volume of sea floor $f^{\prime}$ at $t_{2}$ : 


$$
e:\left(F \times\left\{t_{1}\right\}\right) \rightarrow\left(F \times\left\{t_{2}\right\}\right)
$$

The function $r$ determines the sea floor destruction, assigning to some volume of sea floor $f$ at $t_{1}$ a smaller volume of sea floor $f^{\prime}$ at $t_{2}: r:\left(F \times\left\{t_{1}\right\}\right) \rightarrow\left(F \times\left\{t_{2}\right\}\right)$.

The seismic activity is represented by the function $h$, which assigns to a certain volume of lithosphere and temporal instant a frequency of vibration as an element of $\mathrm{R}^{+}: h$ : $U \times T \rightarrow \mathrm{R}^{+}$.

The movement of the tectonic plates is represented by means of the function $g$. Under it, each volume corresponding to a tectonic plate and a certain spatial region in $t_{1}$ are assigned the same volume of tectonic plates and a different spatial region in $t_{2}$.

$$
g:\left(O \times S \times\left\{t_{1}\right\}\right) \rightarrow\left(O \times S \times\left\{t_{2}\right\}\right)
$$

The function $q$ determines the kinetic friction between volumes of continents, by assigning to each couple of neighbouring volumes and a certain temporal instant a friction coefficient: $q: C \times C \times T \rightarrow \mathrm{R}^{+}$.

Similarly, under the function $n$ each couple of neighbouring volumes corresponding to tectonic plates and a certain temporal instant are assigned a friction coefficient: $n$ : $O \times O \times T \rightarrow \mathrm{R}^{+}$.

The structures contained in TEC's class of potential models must satisfy the typifications and characterizations mentioned above, thereby forming tuples like the following: $<L, U, T, S, O, C, M, F, \mathrm{R}^{+}, d, P, I, e, r, h, g, k, q, n>$.

\subsubsection{TEC's Laws}

As usual, axiom (1) simply states that TEC's class of actual models is a subset of its class of potential models. TEC's specific content is unfolded in seven fundamental laws.

Axiom (4) establishes that sea floor spreading and sea floor destruction are connected to the movement of tectonic plates, and also that the amount of sea floor spreading is approximately the same as the amount of sea floor destruction. Put formally:

For all $o \in O$ there are some $f, f^{\prime} \in F$ such that:

(4i) if $e\left(<f, t_{2}>\right)>f$ at $t_{1}$ and $r\left(<f^{\prime}, t_{2}>\right)<f^{\prime}$ at $t_{1}$, then there is a $<o, s^{\prime}, t_{2}>\epsilon$ $\operatorname{Codom}(g)$

(4ii) for all $\left(<f, t_{1}>\right) \in \operatorname{Dom}(e)$ and $\left(<f^{\prime}, t_{1}>\right) \in \operatorname{Dom}(r): e\left(<f, t_{2}>\right) \approx r\left(<f, t_{2}>\right)$

In axiom (5), continental movement is explained on the basis of the movement of tectonic plates, which causes a variation in the positions of continents. That is:

For all $\left\langle c, s, t_{1}\right\rangle \in \operatorname{Dom}(d)$ there is a $\left.<o, s, t_{1}\right\rangle \in \operatorname{Dom}(g)$ such that: if (4) is the case, then $d\left(<c, s, t_{1>}\right)=<c, s^{\prime}, t_{2}>$

Axiom (6) asserts that mountains form due to kinetic friction either between continents or between continents and sea floors. Formally expressed:

For all $m \in M, c, \mathrm{c}^{\prime} \in C$, either:

(6i) if $\left.q\left(<c, c^{\prime}, t_{1}\right\rangle\right)=i \neq 0$ and $\left.q\left(<c, c^{\prime}, t_{2}\right\rangle\right)=i^{\prime}$ with $\left.i^{\prime}\right\rangle i$, then, neither $m P c$ nor $m P c^{\prime}$ at $t_{1}$, and $m P c$ or $m P c^{\prime}$ at $t_{2}$,

(6ii) there is some $f \in F$ such that if $\left.k\left(<c, f, t_{1}\right\rangle\right)=i \neq 0$ and $\left.k\left(<c, f, t_{2}\right\rangle\right)=i$ ' with $i$ ' $>i$, then, neither $m P c$ nor $m P f$ at $t_{1}$, and $m P c$ or $m P f$ at $t_{2}$

In axiom (7), every part of a continent and of a sea floor is required to be part of some tectonic plate, although not all of them are part of the same one. That is: 
For all $c, f \in U$ there are some $o, o$ ' $\in O$ such that: $c P o$ or $c P o$ ' and $f P o$ or $f P o$, and it is not the case that there is an $o \in O$ such that for all c, $f \in C: c P o$ and $f P o$

Finally, in axiom (8), seismic activity is accounted for on the basis of kinetic friction between tectonic plates. Put formally:

For all $u \in \operatorname{Dom}(h)$ there is some $\left\langle o, o^{\prime}, t_{1}\right\rangle \in \operatorname{Dom}(n)$ such that: if $\left.n\left(<o, o^{\prime}, t_{2}\right\rangle\right) \neq$ 0 then $h\left(<u, t_{2}>\right) \neq 0$.

\subsubsection{TEC's Empirical Framework}

TEC's empirical concepts, i.e., those determining its class of partial potential models and independent of its fundamental laws, can be represented as follows:

$\mathbf{M}_{\mathbf{p p}}$ (TEC): $y$ is a partial potential model of plate tectonics $\left(y \in \mathbf{M}_{\mathbf{p p}}(\mathbf{T E C})\right)$ iff there exists an $x$ such that $x=<L, U, T, S, O, C, M, F, \mathrm{R}^{+}, d, P, I, e, r, h, g, k, q, n>\in$ $\mathbf{M}_{\mathbf{p}}$ (TEC) and $y=<L, U, T, S, C, M, F, \mathrm{R}^{+}, P, I, h>$

The question of theoreticity concerns $L, U, T, S, O, C, M, F, \mathrm{R}^{+}, d, P, I, e, r, h, g, k, q$, $n$. TEC includes all DRIFT-non-theoretical concepts, namely: $L, U, T, S, C, M, F, P, I$. In addition to them, TEC includes a new TEC-non-theoretical concept, that of seismic activity $(h)$, whose determination does not involve TEC's laws, but merely relying on observations obtained from the use of instruments such as seismographs or seismometers.

As for TEC-theoretical concepts, it is worth noting that $d$, which is the main DRIFTtheoretical concept, is included in TEC as a TEC-theoretical concept as well, since, according to TEC's upholders, determining $d$ requires the assumption that some moving tectonic plates underlie the continents (axiom 5). As emphasized by numerous scholars (Gould 1977; Laudan 1978; Marvin 2001; Ruse 1981/1989), continental movement was not unanimously regarded as an acceptable postulated phenomenon until TEC's proponents offered a more detailed and better empirically grounded account of such phenomenon. Something similar happens with $k$ (the concept of kinetic friction between continents and sea floors), which is both DRIFT-theoretical and TEC-theoretical. While determining $k$ within DRIFT always presupposes applying axioms (4) and (5) from this theory, determining $k$ within TEC always requires assuming axioms (5) and (6ii) from TEC. Thus, even if the concept of kinetic friction between continents and sea floors is included in both theories, its determination involves the assumption of different axioms depending on what theory we are applying. If we apply DRIFT, a necessary assumption is the motion of continents, whereas if we apply TEC, a necessary assumption is the motion of tectonic plates, since otherwise continental drift would not be assumed. Yet, continental drift is a shared presupposition in both theories when $k$ needs to be determined. More precisely, in determining $k$, continental drift is the main presupposition held by DRIFT's advocates, and is only part of the main presupposition held by TEC's proponents. ${ }^{8}$ Here again it becomes evident that overcoming the long-term skepticism aroused by continental drift as postulated from DRIFT did not only involve new independent evidence supporting

\footnotetext{
$\overline{8}$ Kinetic friction is thus one of the parameters common to both DRIFT and TEC. To be more faithful to the historical background, it would be necessary to distinguish between viscous friction and rigid friction. Wegener postulated a viscous friction that would operate between continents and sea floors, since he conjectured that there was a process of plastic yielding of the oceanic "sima" and likewise a process of plastic folding (mountain building) of the continental "sial" upon lateral compression. On the other hand, by adding the concept of rigid plate, TEC also contains viscous friction both at the plates' bottom (convection as a plate driving force) and between continents as well as rigid friction at the plates' margins (as the cause of earthquakes). However, for reasons of parsimony, the above distinctions have not been included in the formal reconstruction of both theories.
} 
TEC, but also an essential connection between the postulated phenomena of continental drift and tectonic motion.

Among the other TEC-theoretical concepts, $e, r, O, g, q$ and $n$, none are shared with previous geological theories. The notion of tectonic plate and that of tectonic plate's movement are specific of TEC and they refer respectively to a kind of entity and property whose determination always depends on axioms (4) and (5). On the other hand, neither sea floor spreading $(e)$, nor sea floor destruction $(r)$ can be defined unless axiom (4) is endorsed. Characterizing the notion of kinetic friction, either between continents, sea floors and continents or between tectonic plates, requires endorsing axioms (6) and (8) respectively.

\subsection{The Embedding of Continental Drift into Plate Tectonics}

Let us move now to the question of the connection between both theories. The fact that there is an inter-theoretical relation between TEC and DRIFT is manifest not only in their mutual appeal to the concept of continental movement, but also in both theories sharing some (parts) of their fundamental laws. In particular, axioms 2 and 3 are shared by both, and axioms 4 and 5 from DRIFT are part of axioms 5 and 6 in TEC. Note that the idea that this inter-theoretical relation is one of (strong) theoretization should be discarded, since, as already explained at the end of Sect. 3, DRIFT-theoretical concepts are not included in TEC as TEC-non-theoretical ones but are instead presupposed in TEC-theoretical ones. There are two main reasons to come to this conclusion. First, Wegener's theory was generally not accepted by the scientific community at the time when TEC developed, and consequently the motion of continents was far from being regarded as a well-established empirical in need for explanation. Second, continental lateral motion is part of the complex conjecture put forward by TEC's upholders in order to explain a wide variety of empirical phenomena, some of them also targeted by Wegener's conjecture. In other words, DRIFT's explanatory resources are kept or embedded in TEC also as explanatory resources. Therefore, as suggested in the first section and argued next, the relation between both theories is best captured in terms of embedding.

In illustrating how Definition 2.2. applies to this case, the notion of theory-net will be replaced by that of theory-element, for, as pointed out earlier, no specializations have been distinguished in the previous reconstructions, which were limited to the respective basic theory-elements of DRIFT and TEC. Given the nature of the conditions specified in the definition of embedding, nothing essential is lost in making use of this option. According to such definition, for DRIFT to be embedded into TEC, two conditions should be met, namely:

1. That to every successful model in DRIFT there corresponds a set-theoretic construct in TEC that results from performing operation $\Theta$ over the components of such model and constitutes a successful model in TEC,

2. That for every successful application in DRIFT there is a set-theoretic construct in TEC that results from performing operation $\Theta$ over the components of such application and constitutes a successful application in TEC.

Intuitively, the embedding at the theoretical level is captured by the first condition, and the requirement for embedding at the empirical level is expressed by the second condition. If we use the notation introduced in Sect. 2, we can paraphrase the conditions as stating that every successful model in DRIFT should be an (approximative) echeloned partial substructure of some successful model in TEC, and every successful intended application 
in DRIFT should be an (approximative) echeloned partial substructure of some successful intended application in TEC.

In the interest of simplicity, the fit of the continental edges and mountain formation will be regarded as DRIFT's main (paradigmatic) intended applications, and these together with seismic activity as TEC's main (paradigmatic) ones. Now, as we know from the previous sections, DRIFT's successful models $\mathrm{x}_{\mathrm{D}}$ are tuples of the following kind: $<L, U, T, S, C, M$, $F, \mathrm{R}^{+}, d, k, P, I>$, satisfying the typifications, characterizations and laws made explicit above, and whose empirical substructures correspond to DRIFT's paradigmatic intended applications. Similarly, TEC's successful models $\mathrm{x}_{\mathrm{T}}$ are structures that can be represented as follows: $<L, U, T, S, O, C, M, F, \mathrm{R}^{+}, d, P, I, e, r, h, m, k, q, n>$, meeting all the corresponding conditions and whose empirical substructures correspond to TEC's paradigmatic intended applications. Taking into account that all successful applications of DRIFT's modelsregarding the fit of continental coastlines and mountain building- are also successful applications of TEC's models, it seems obvious that the empirical side of condition (1) is met, i.e., there is a successful model in TEC for every successful model in DRIFT.

Let us now focus on the formal side of the first condition (1). To see whether this condition is fully met, we need to apply Definition 1.5 and check whether every $\mathrm{x}_{\mathrm{D}}$ is an (approximative) echeloned partial substructure of some $x_{T}$, since in that case $x_{D}$ is an echeloned partial substructure $\mathrm{x}_{\mathrm{T}}\left(\mathrm{x}_{\mathrm{D}} \eta \mathrm{x}_{\mathrm{T}}\right)$. According to the definition of echeloned partial substructure (1.5.), then we must determine whether for all $\mathrm{x}_{\mathrm{Di}} \in \mathrm{x}_{\mathrm{D}}$ there is some $\mathrm{x}_{\mathrm{Tk}} \hat{\in} \mathrm{x}_{\mathrm{T}}$ such that $\mathrm{x}_{\mathrm{Di}} \in \Theta\left(\mathrm{x}_{\mathrm{Tk}}\right)$.

In the present case, however, there is no need to operate with partial substructures $\left(\mathrm{x}_{\mathrm{Di}}\right)$ of DRIFT's successful models $\left(\mathrm{x}_{\mathrm{D}}\right)$, for the whole structures from DRIFT are preserved in TEC. On the other hand, the relevant partial substructure of $\mathrm{x}_{\mathrm{T}}$ looks like the following ${ }^{9}$ :

$$
x_{T k=}\left\langle L, U, T, S, C, M, F, R^{+}, d, k, P, I\right\rangle
$$

It should be noted that $\mathrm{x}_{\mathrm{Tk}}$ is simply the result of removing some of the TEC-theoretical components from $\mathrm{x}_{\mathrm{Tk}}$. This operation provides a partial substructure of $\mathrm{x}_{\mathrm{T}}$ that is identical to $\mathrm{x}_{\mathrm{D}}=\left\langle L, U, T, S, C, M, F, \mathrm{R}^{+}, d, k, P, I>\right.$. So we arrive at the following result:

for all $\mathrm{x}_{\mathrm{D}}$ there is some $\mathrm{x}_{\mathrm{Tk}} \hat{\in} \mathrm{x}_{\mathrm{T}}$ such that $\mathrm{x}_{\mathrm{D}}=\mathrm{x}_{\mathrm{Tk}}$, thus, by Definition 1.2., $\mathrm{x}_{\mathrm{D}} \hat{\in} \mathrm{x}_{\mathrm{T}}$.

That is, every successful model in DRIFT is a partial substructure of a successful model in TEC, as every component from the former is also a component from the latter, while the reverse does not hold. This result is even stricter in terms of preservation than what was required in Definition 2.2. for embedding, since what is preserved from the previous theory is not just echeloned partial substructures, not even partial substructures, but whole structures.

As for condition (2) of embedding, similar considerations apply. As pointed out earlier, all of DRIFT's successful applications $\left(\mathrm{y}_{\mathrm{D}}\right)$ are also TEC's successful applications $\left(\mathrm{y}_{\mathrm{T}}\right)$. Again at the level of successful applications it is not necessary to operate with DRIFT's partial substructures $\left(\mathrm{y}_{\mathrm{Di}}\right)$, for the whole structures from DRIFT's class of successful applications, as well as the specific interpretations of such structures, are preserved in TEC. Here the relevant partial substructure of $\mathrm{y}_{\mathrm{T}}$ would be the following:

$$
y_{T k}=<L, U, T, S, C, M, F, R^{+}, P, I>
$$

This partial substructure results simply from removing the component $h$ (i.e., the function for seismic activity) from TEC-non theoretical components. As happened at the theoretical

${ }^{9}$ In ' $\mathrm{x}_{\mathrm{Di}}$ ' ' $\mathrm{i}$ ' is a sub-index of a sub-index (' $\mathrm{T}$ '). The same goes for ' $\mathrm{x}_{\mathrm{Tk}}$ ', ' $\mathrm{y}_{\mathrm{Di}}$ ', $\mathrm{y}_{\mathrm{Tk}}$ '. 
level, this operation provides a partial substructure of $\mathrm{y}_{\mathrm{T}}$ that is identical to $\mathrm{y}_{\mathrm{D}}=\langle L, U, T$, $S, C, M, F, \mathrm{R}^{+}, P, I>$. So we obtain an analogous result at the non-theoretical level:

for all $\mathrm{y}_{\mathrm{D}}$ there is some $\mathrm{y}_{\mathrm{Tk}} \hat{\in} \mathrm{y}_{\mathrm{T}}$ such that $\mathrm{y}_{\mathrm{D}}=\mathrm{y}_{\mathrm{Tk}}$, thus, by Definition 1.2., $\mathrm{y}_{\mathrm{D}} \hat{\in} \mathrm{y}_{\mathrm{T}}$. This means that every successful application in DRIFT is a partial substructure of a successful application in TEC, given that every component from the former is also a component from the latter, and not the other way around. Again in terms of preservation, this result goes further than what was contemplated in the definition of embedding, for whole empirical structures from DRIFT, as well as the specific interpretations of such structures, as opposed to either echeloned partial substructures or partial substructures, have been preserved in TEC.

\section{Conclusions}

The present study has shown that the relation between drift theory and plate tectonics fulfills the conditions for embedding. All theoretical concepts from drift theory are presupposed in some theoretical concepts from plate tectonics, and all empirical concepts of the former are shared by the latter. Furthermore, all the successful paradigmatic applications of continental drift are also successful applications of plate tectonics. As seen in the previous discussion, there is no semantic incommensurability between both theories, since they are not only conceptually compatible but also complementary. The taxonomic changes identified by Thagard thus result in an addition of properties ascribed to continents and sea floors, rather than in conflicting conceptualizations.

This approach also rules out the possibility of regarding plate tectonics as a theoretization of drift theory - something frequently suggested from standard historical accounts-, since the theoretical concepts of continental drift are not included among the non-theoretical ones of plate tectonics. In fact, the very concept of continental drift is included in plate tectonics as a theoretical concept as well, since, according to the upholders of the latter, determining continental motion requires the assumption that some moving tectonic plates underlie the continents. This conclusion is in agreement with the view of numerous scholars (Gould 1977; Laudan 1978; Marvin 2001; Ruse 1981/1989), who have emphasized that continental movement was not unanimously regarded as real phenomenon until the proponents of plate tectonics offered a more detailed and better empirically grounded framework to integrate this phenomenon. The present reconstruction of geological theories shows that the motion of continents was not part of the intended explanandum of plate tectonics but rather part of its explanans, for both theories postulate this kind of motion to account for some other facts, like mountain building or the fit of continental coastlines.

Finally, the embedding of continental drift into plate tectonics undermines the Kuhnian idea that innovative theories embedding previous ones constitute a hardly existing instance of scientific development. Here, under the label "geological revolution", we have a salient historical case of cumulative progress across theory change.

Acknowledgements I am thankful to José Díez for helpful comments on an earlier version of this paper. Thanks also to the participants in the 8th and 9th editions of the Latin American Conference on Structuralist Metatheory (Mexico DF 2012, Barcelona 2014), where I had the chance to get a valuable feedback on different aspects of this work. Thanks specially to Matías Aimino and Adriana Gonzalo fo their illuminating discussion on the principle of isostasy. My gratitude also to Jurgen Strehlau for his useful suggestions to improve my analysis of the historical case. Finally, this paper has greatly benefited from comments by three anonymous referees. This research was financially supported by the research projects "Pragmatics as the Driven Force Behind the Study of Semantic Flexibility: Conversational Contexts and Theoretical Contexts" 
(FII 2012-33881, Spanish Ministry of Economy and Competitiveness) and "Models and Theories in Physical, Biological and Social Sciences” (PICT-2014-1741, ANPCyT, Argentina).

\section{References}

Balzer, W., Moulines, C. U., \& Sneed, J. D. (1987). An architectonic for science: The structuralist program. Dordrecht: Reidel.

Barrell, J. (1914). The strength of the Earth's crust. Journal of Geology, 22(4), 289-314.

Cohen, I. B. (1985/2001). "Continental Drift and Plate Tectonics: A Revolution in the Earth Science", Revolution in Science. Cambridge: Harvard University Press, ch. 29: 446-466.

Daly, R. A. (1940). Strength and Structure of the Earth. New York: Prentice-Hall.

Falguera, J. L., \& Donato Rodríguez, X. (2015). Incommensurability, Comparability, and Non-reductive Ontological Relations. Journal for the General Philosophy of Science, . doi:10.1007/s10838-014-92753.

Feyerabend, P. K. (1962/1981). "Explanation, Reduction, and Empiricism", Realism, Rationalism and Scientific Method. Philosophical Papers, vol. I. Cambridge: Cambridge University Press.

Frankel, H. (1979). The career of continental drift theory: An application of imre lakatos' analysis of scientific growth to the rise of drift theory. Studies in History and Philosophy of Science, 10(1), 21-66.

Frisch, W., Meschede, M., \& Blakey, R. (2011). Plate tectonics: Continental drift and mountain building. Heidelberg: Springer.

Gould, S. J. (1977). "The Validation of Continental Drift", Ever Since Darwin: Reflections in Natural History. New York: Norton, chapter 5, section 20: 160-167.

Kuhn, T. S. (1962/1970). The Structure of Scientific Revolutions. Chicago, The University of Chicago Press.

Kuhn, T. S. (1969/1970). "Postscript-1969", in The Structure of Scientific Revolutions. Chicago, The University of Chicago Press: 182-191.

Kuhn, T. S. (1983). Commensurability, comparability, communicability. Philosophy of Science Association, II, 669-688.

Laudan, R. (1978). "The recent revolution in geology and kuhn's theory of scientific change", PSA: Proceedings of the Biennial Meeting of the Philosophy of Science Association, vol. 2, Symposia and Invited Papers: 227-239.

Marvin, U. B. (2001). Reflections on the American rejection of continental drift. Metascience, 10, $208-217$.

Moulines, C. U. (1996). "Zur Typologie wissenschaftlicher Entwicklung nach strukturalistischer Deutung". (In Hubig, C. \& H. Poser (eds.), Cognitio Humana-Dynamik des Wissens und der Werte, XVII, Leipzig: Institut für Philosophie der Universität Leipzig, 397-410.

Moulines, C. U. (2002). Introduction: Structuralism as a program for modelling theoretical science. Synthese, $130,1-11$.

Moulines, C. U. (2010). "The crystallization of Clausius's phenomenological thermodynamics". (In Andreas Hüttemann \& Gerhard Ernst (eds.), Time, Chance, and Reduction: Philosophical Aspects of Statistical Mechanics, Cambridge: Cambridge University Press: 139-158.

Moulines, C. U. (2011). Cuatro tipos de desarrollo teórico en las ciencias empíricas. Metatheoria, 1(2), 11-27.

Moulines, C. U. (2014). Intertheoretical relations and the dynamics of science. Erkenntnis, 79(Supplement 8), 1505-1519.

Oreskes, N. (2003). "From Continental Drift to Plate Tectonics". (In Naomi Oreskes \& Homer Le Grand (eds.) Plate Tectonics: An Insiders' History of the Modern Theory of the Earth, Boulder: Westview Press, chapter 1: 3-27.

Ruse, M. (1981/1989). "What Kind of Revolution Occurred in Geology", The Darwinian Paradigm, Essays on Its History, Philosophy and Religious Implications. London, Routledge, ch. 3: 55-89.

Thagard, P. (1992). Conceptual revolutions. Princeton: Princeton University Press.

Van Fraassen, B. (1980). The scientific image. Oxford: Oxford University Press.

María Caamaño-Alegre is associate professor at the Department of Philosophy at the University of Valladolid, Spain. She holds a Ph.D. in philosophy from the University of Santiago de Compostela (2004, Spain) on the incommensurability of scientific theories. Her fields of interest include general philosophy of science, topics within the intersection between philosophy of science and philosophy of language, methodology of science and theory assessment. She has recently published on the problems of non-refuting anomalies and framing effects. 